\title{
APPLICATION OF THE WAVE FINITE ELEMENT METHOD TO MULTI-SPAN BRIDGES
}

\author{
G. Paratore ${ }^{2}$, T. Hoang ${ }^{1}$, G. Foret ${ }^{1}$, M.P. Limongelli ${ }^{3}$, and D. Duhamel ${ }^{1}$ \\ ${ }^{1}$ Ecole des Ponts ParisTech \\ Champs sur Marne, France \\ e-mail: \{denis.duhamel,tien.hoang,gilles.foret\}@enpc.fr \\ ${ }^{2}$ Politecnico di Torino \\ Torino, Italy \\ e-mail: gabriele.paratore@ studenti.polito.it \\ ${ }^{3}$ Politecnico di Milano \\ Milano, Italy \\ e-mail: mariagiuseppina.limongelli@polimi.it
}

Keywords: Wave Finite Element, support, bridge, dynamics.

\begin{abstract}
The Wave Finite Element (WFE) method is based on wave propagation in periodic structures. Starting from a Finite Element (FE) analysis of a single period (sub-structure) we are able to derive the dynamic behaviour relative to the entire structure. Thanks to a reduction in the degrees of freedom (dofs) of the system and by decomposing the response of the structure on a wave basis, the calculation time is considerably reduced compared to the classic FEM. Numerous structures have been solved with this method but it can not deal easily on the boundary conditions. In this study, we develop a technique of WFE to deal with more general cases of structures constrained in a arbitrary manner as a multiple supported bridge. By using the WFE method, the vectors of dofs and loads will be decomposed on the wave basis in function of loads and reaction forces of the supports. Then, by substituting the boundary condition in this wave decomposition, we obtain a relation between the reaction forces and the loads which permits to calculate the structure response. The numerical applications show that the WFE and FEM agree well and the new method permits to reduce significantly the calculation time.
\end{abstract}




\section{INTRODUCTION}

The Wave Finite Element (WFE) is a powerful numerical method that allows to drastically reduce the number of degrees of freedom of a structural model providing a great advantage in terms of computational time. This method has been developed for periodic structures where the geometry repeats itself in a certain direction, and is based on wave propagation. The objective is to compute the wave modes which constitute a base that, similarly to the eigen modes often used in the dynamic analysis, can be used to decompose the structural response in terms of forces and displacements at the considered degrees of freedom. The wave modes are characteristics of the elementary periodic element therefore they can be computed by solving an eigenvalues problem consistently formulated for this element. According to the approach proposed by Duhamel et al. $[1,2,3]$ the periodic element can be modelled using the conventional finite element method to retrieve its mass, stiffness and damping matrices. In this approach, these matrices are then used to obtain the dynamic stiffness of the cell in the frequency domain and the theory of periodic structure is used to build the dynamic stiffness matrix of the whole structure. Once the wave base has been determined for the period of the structure and the dynamic problem formulated in terms of this base, the solution of the dynamic equation can be carried out by imposing the boundary conditions. This approach has been followed in Hoang et al. [4] that proposed the wave approach, based on the computation of the dynamic response as the sum of different wave contributions generated by the forces acting on the structure. In previous studies $[4,5]$, the WFE has been applied considering constrains applied only at the ends of the structure or included inside the periodic element, as for the case of railway tracks. The objective of this paper is to extend the application of the WFE to the study of the dynamic response of multi-supported periodic structures, including the possibility to account for different types of boundary conditions. The extension proposed in this paper allows to analyse structures, such as bridges, whose supports may be of different types and are not necessarily equally spaced. The paper is structured in four sections. The second is dedicated to the introduction of the WFE starting from the type of structures considered in the study and the approaches of resolution. The third section contains the original part of this paper dealing with the analytical formulation of the WFE of a periodic structure with multiple intermediate supports. Finally, in the fourth section the numerical results will be presented with reference to a number of case studies.

\section{WAVE FINITE ELEMENT}

\subsection{Discrete formulation}

A periodic system consists of a number of identical elements, in terms of geometric shape, physical properties, boundary conditions, and connections with other substructures, coupled together to form the whole system, see Figure (1). Using the FE method, the generic element is modelled and two types of nodes can be defined: internal nodes and boundary nodes. The boundary nodes are in turn divided into left nodes and right nodes with $d$ degrees of freedom (dofs) for each side. The kinematic behaviour of the system is described by the column vector of nodal displacements q which number of components is equal to the total number of dofs of the substructure. On the other hand, the respective nodal forces are described by the column vector $\mathbf{F}$. Their relation is given by the discrete equation of motion:

$$
\left(\mathbf{K}+i \omega \mathbf{C}-\omega^{2} \mathbf{M}\right) \mathbf{q}(\omega)=\mathbf{D}(\omega) \mathbf{q}(\omega)=\mathbf{F}(\omega)
$$




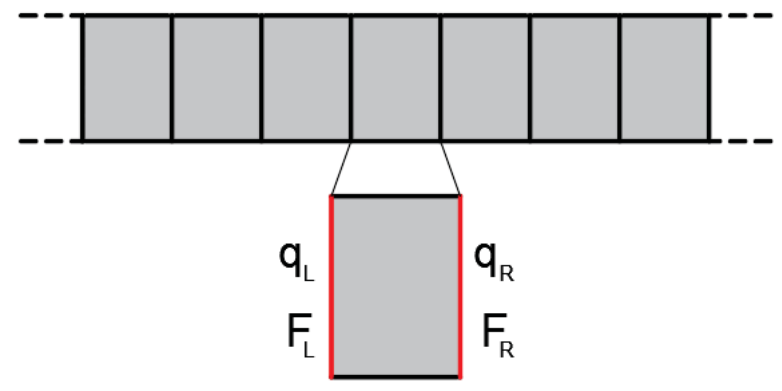

Figure 1: General substructure.

where $\mathbf{D}$ is the dynamic stiffness matrix (DSM), and $\mathbf{M}, \mathbf{C}$ and $\mathbf{K}$ are respectively the Mass, Dumping and Stiffness Matrix. The dynamic equation can be rewritten in the expanded form in order to separate the degrees of freedom into left (L), inner (I) and right (R) ones.

$$
\left[\begin{array}{lll}
\mathbf{D}_{L L} & \mathbf{D}_{L I} & \mathbf{D}_{L R} \\
\mathbf{D}_{I L} & \mathbf{D}_{I I} & \mathbf{D}_{I R} \\
\mathbf{D}_{R L} & \mathbf{D}_{R I} & \mathbf{D}_{R R}
\end{array}\right]\left[\begin{array}{c}
\mathbf{q}_{L} \\
\mathbf{q}_{I} \\
\mathbf{q}_{R}
\end{array}\right]=\left[\begin{array}{c}
\mathbf{F}_{L} \\
\mathbf{F}_{I} \\
\mathbf{F}_{R}
\end{array}\right]
$$

Writing the inner degrees of freedom $\mathbf{q}_{I}$ in function of the boundary dofs $\mathbf{q}_{R}$ and $\mathbf{q}_{L}$, we can reduce the inner nodes in order to obtain a condensed form of the dynamic stiffness matrix.

$$
\left[\begin{array}{l}
\overline{\mathbf{D}}_{L I} \mathbf{F}_{I} \\
\overline{\mathbf{D}}_{R I} \mathbf{F}_{I}
\end{array}\right]+\left[\begin{array}{ll}
\overline{\mathbf{D}}_{L L} & \overline{\mathbf{D}}_{L R} \\
\overline{\mathbf{D}}_{R L} & \overline{\mathbf{D}}_{R R}
\end{array}\right]\left[\begin{array}{l}
\mathbf{q}_{L} \\
\mathbf{q}_{R}
\end{array}\right]=\left[\begin{array}{l}
\mathbf{F}_{L} \\
\mathbf{F}_{R}
\end{array}\right]
$$

Where,

$$
\begin{aligned}
& \overline{\mathbf{D}}_{L L}=\mathbf{D}_{L L}-\mathbf{D}_{L I} \mathbf{D}_{I I}^{-1} \mathbf{D}_{I L}, \overline{\mathbf{D}}_{L R}=\mathbf{D}_{L R}-\mathbf{D}_{L I} \mathbf{D}_{I I}^{-1} \mathbf{D}_{I R}, \overline{\mathbf{D}}_{R L}=\mathbf{D}_{R L}-\mathbf{D}_{R I} \mathbf{D}_{I I}^{-1} \mathbf{D}_{I L} \\
& \overline{\mathbf{D}}_{R R}=\mathbf{D}_{R R}-\mathbf{D}_{R I} \mathbf{D}_{I I}^{-1} \mathbf{D}_{I R}, \overline{\mathbf{D}}_{L I}=\mathbf{D}_{L I} \mathbf{D}_{I I}^{-1}, \overline{\mathbf{D}}_{R I}=\mathbf{D}_{R I} \mathbf{D}_{I I}^{-1}
\end{aligned}
$$

Considering two consecutive substructures $(n)$ and $(n+1)$, two fundamental conditions must be always satisfied: continuity of the displacement along the junction $\mathbf{q}_{R}^{(n)}=\mathbf{q}_{L}^{(n+1)}$, equilibrium of the forces acting in the junction: $\mathbf{F}_{R}^{(n)}+\mathbf{F}_{L}^{(n+1)}=\mathbf{F}_{B}^{(n)}$. These equations can be manipulated in order to write the terms regarding substructure $(n+1)$ in function of those regarding the preceding substructure $(n)$.

$$
\left[\begin{array}{c}
\mathbf{q}_{L}^{(n+1)} \\
-\mathbf{F}_{L}^{(n+1)}
\end{array}\right]=\mathbf{S}\left[\begin{array}{c}
\mathbf{q}_{L}^{(n)} \\
-\mathbf{F}_{L}^{(n)}
\end{array}\right]+\left[\begin{array}{c}
\overline{\mathbf{D}}_{q I} \mathbf{F}_{I}^{(n)} \\
\overline{\mathbf{D}}_{f I} \mathbf{F}_{I}^{(n)}-\mathbf{F}_{B}^{(n)}
\end{array}\right]
$$

Where, $\mathbf{S}$ is the transfer matrix given by:

$$
\mathbf{S}=\left[\begin{array}{cc}
-\overline{\mathbf{D}}_{L R}^{-1} \overline{\mathbf{D}}_{L L} & -\overline{\mathbf{D}}_{L R}^{-1} \\
\overline{\mathbf{D}}_{L R}-\overline{\mathbf{D}}_{R R} \overline{\mathbf{D}}_{L R}^{-1} \overline{\mathbf{D}}_{L L} & -\overline{\mathbf{D}}_{R R} \overline{\mathbf{D}}_{L R}^{-1}
\end{array}\right]
$$

with $\overline{\mathbf{D}}_{q I}=-\overline{\mathbf{D}}_{L R}^{-1} \overline{\mathbf{D}}_{L I}$ and $\overline{\mathbf{D}}_{f I}=\overline{\mathbf{D}}_{R I}-\overline{\mathbf{D}}_{R R} \overline{\mathbf{D}}_{L R}^{-1} \overline{\mathbf{D}}_{L I}$.

The nodal displacements $\mathbf{q}^{(n)}$ and the nodal forces $\mathbf{F}^{(n)}$, together describe the state of the substructure. For this reason we define the state vector $\mathbf{u}^{(n)}$ of the substructure $(n)$ and $\mathbf{b}^{(n)}$ the vector of external loads acting on the substructure $(n)$ as:

$$
\mathbf{u}^{(n)}=\left[\begin{array}{c}
\mathbf{q}_{L}^{(n)} \\
-\mathbf{F}_{L}^{(n)}
\end{array}\right], \quad \mathbf{b}^{(n)}=\left[\begin{array}{c}
\overline{\mathbf{D}}_{q I} \mathbf{F}_{I}^{(n)} \\
\overline{\mathbf{D}}_{f I} \mathbf{F}_{I}^{(n)}-\mathbf{F}_{B}^{(n)}
\end{array}\right]
$$


Equation (4) can be written in a more synthetic form:

$$
\mathbf{u}^{(n+1)}=\mathbf{S u}^{(n)}+\mathbf{b}^{(n)}
$$

This last equation represents the relation between the generic substructure $(n)$ and its next substructure $(n+1)$ by means of the transfer matrix $\mathbf{S}$. This leads to:

$$
\mathbf{u}^{(n)}=\mathbf{S}^{n-1} \mathbf{u}^{(1)}+\sum_{k=1}^{n-1} \mathbf{S}^{n-k-1} \mathbf{b}^{(k)}
$$

Equation (8) represents the relation between the state vector of the first substructure and the state vector of the substructure $(n)$. One also has

$$
\mathbf{u}^{(N+1)}=\mathbf{S}^{N-n+1} \mathbf{u}^{(n)}+\sum_{k=n}^{N} \mathbf{S}^{N-k} \mathbf{b}^{(k)}
$$

\subsection{Wave formulation}

The wave modes are the eigenvectors solution of

$$
\mathbf{S}\left[\begin{array}{c}
\mathbf{q}_{L} \\
-\mathbf{F}_{L}
\end{array}\right]=\lambda\left[\begin{array}{c}
\mathbf{q}_{L} \\
-\mathbf{F}_{L}
\end{array}\right]
$$

Hence, each eigenvalue of the matrix $\mathbf{S}$ provides a propagation constant $\lambda_{i}$ while the eigenvector $\Phi_{i}$ represents the wave shapes, namely the way in which the substructure deforms. For each eigenvalue $\lambda_{i}$ of eigenvector $\Phi_{i}$, there is an eigenvalue $1 / \lambda_{i}$ associated to an eigenvector denoted $\Phi_{i}^{*}$.

The matrix $\mathbf{S}$ is symplectic meaning that ${ }^{t} \mathbf{S J S}=\mathbf{J}$ with $\mathbf{J}=\left[\begin{array}{cc}\mathbf{O} & \mathbf{I} \\ -\mathbf{I} & \mathbf{O}\end{array}\right]$. The orthogonality properties are such that ${ }^{t} \boldsymbol{\Phi}^{*} \mathbf{J} \boldsymbol{\Phi}=\mathbf{I},{ }^{t} \boldsymbol{\Phi}^{*} \mathbf{J} \boldsymbol{\Phi}^{*}={ }^{t} \boldsymbol{\Phi} \mathbf{J} \boldsymbol{\Phi}=\mathbf{O}$ with $\boldsymbol{\Phi}=\left[\Phi_{1}, \ldots, \Phi_{d}\right]$.

We can decompose the state vector $\mathbf{u}^{(n)}$ and the load vector $\mathbf{b}^{(n)}$ in the wave base as a combination of positive and negative waves:

$$
\begin{aligned}
\mathbf{u}^{(n)} & =\boldsymbol{\Phi} \mathbf{Q}^{(n)}-\boldsymbol{\Phi}^{*} \mathbf{Q}^{*(n)} \\
\mathbf{b}^{(n)} & =\Phi \mathbf{Q}_{B}^{(n)}-\Phi^{*} \mathbf{Q}_{B}^{*(n)}
\end{aligned}
$$

Where $\mathrm{Q}^{(n)}$ and $\mathrm{Q}^{*(n)}$ represent the vectors of wave amplitude of the positive-going and negativegoing waves. The terms $\mathbf{Q}_{B}^{(n)}$ and $\mathrm{Q}_{B}^{*(n)}$ are the vectors of wave amplitude generated by the external loads $\mathbf{F}_{I}^{(n)}$ and $\mathbf{F}_{B}^{(n)}$. The wave amplitudes of the external loads can be derived from equation (11), by multiplying both sides by $\boldsymbol{\Phi}^{* T} \mathbf{J}$ as follow:

$$
\begin{aligned}
\boldsymbol{\Phi}^{* T} \mathbf{J} b^{(n)} & =\boldsymbol{\Phi}^{* T} \mathbf{J} \boldsymbol{\Phi} \mathbf{Q}_{B}^{(n)}-\boldsymbol{\Phi}^{* T} \mathbf{J} \boldsymbol{\Phi}^{*} \mathbf{Q}_{B}^{*(n)} \\
& =\mathbf{Q}_{B}^{(n)} \\
& =\left(\boldsymbol{\Phi}_{q}^{* T} \overline{\mathbf{D}}_{f I}-\boldsymbol{\Phi}_{F}^{* T} \overline{\mathbf{D}}_{q I}\right) \mathbf{F}_{I}^{(n)}-\boldsymbol{\Phi}_{q}^{* T} \mathbf{F}_{B}^{(n)}
\end{aligned}
$$

The same thing can be done to compute the value of $\mathbf{Q}_{B}^{*(k)}$. Multiplying both sides by $\boldsymbol{\Phi}^{T} \mathbf{J}$ :

$$
\begin{aligned}
\boldsymbol{\Phi}^{T} \mathbf{J} b^{(n)} & =\boldsymbol{\Phi}^{T} \mathbf{J} \boldsymbol{\Phi} \mathbf{Q}_{B}^{(n)}-\boldsymbol{\Phi}^{T} \mathbf{J} \boldsymbol{\Phi}^{*} \mathbf{Q}_{B}^{*(n)} \\
& =\mathbf{Q}_{B}^{*(n)} \\
& =\left(\boldsymbol{\Phi}_{q}^{T} \overline{\mathbf{D}}_{f I}-\boldsymbol{\Phi}_{F}^{T} \overline{\mathbf{D}}_{q I}\right) \mathbf{F}_{I}^{(n)}-\boldsymbol{\Phi}_{q}^{T} \mathbf{F}_{B}^{(n)}
\end{aligned}
$$




\section{WFE FOR STRUCTURES WITH SEVERAL SUPPORTS}

\subsection{Derivation of the intermediate reactions}

In this section, a technique of WFE will be developed to deal with more general cases of structures constrained in a arbitrary manner as a multiple supported bridge. By convention, the constrain can be placed only in the junction between two substructures. If we consider the reactions as external forces acting on the structure, from the equilibrium of external forces and internal forces we have:

$$
\mathbf{F}_{R}^{(k)}+\mathbf{F}_{L}^{(k+1)}=\mathbf{F}_{e x t}^{(k)}+\mathbf{R}^{(k)}
$$

Hence this time $\mathbf{F}_{B}$ is given by:

$$
\mathbf{F}_{B}^{(k)}=\mathbf{F}_{e x t}^{(k)}+\mathbf{R}^{(k)}
$$

The wave amplitudes can be expressed in function of the external loads $\mathbf{F}_{\text {ext }}^{(k)}$ and reactions $\mathbf{R}^{(k)}$ as follows from (12) and (13):

$$
\begin{gathered}
\mathbf{Q}_{B}^{(k)}=\left(\boldsymbol{\Phi}_{q}^{* T} \overline{\mathbf{D}}_{f I}-\boldsymbol{\Phi}_{F}^{* T} \overline{\mathbf{D}}_{q I}\right) \mathbf{F}_{I}^{(k)}-\boldsymbol{\Phi}_{q}^{* T}\left(\mathbf{F}_{e x t}^{(k)}+\mathbf{R}^{(k)}\right) \\
\mathbf{Q}_{B}^{*(k)}=\left(\boldsymbol{\Phi}_{q}^{T} \overline{\mathbf{D}}_{f I}-\boldsymbol{\Phi}_{F}^{T} \overline{\mathbf{D}}_{q I}\right) \mathbf{F}_{I}^{(k)}-\boldsymbol{\Phi}_{q}^{T}\left(\mathbf{F}_{e x t}^{(k)}+\mathbf{R}^{(k)}\right)
\end{gathered}
$$

The sum (8) can be written as

$$
\Phi \mathbf{Q}^{(n)}-\boldsymbol{\Phi}^{*} \mathbf{Q}^{*(n)}=\mathbf{S}^{n-1}\left(\boldsymbol{\Phi} \mathbf{Q}^{(1)}-\boldsymbol{\Phi}^{*} \mathbf{Q}^{*(1)}\right)+\sum_{k=1}^{n-1} \mathbf{S}^{n-k-1}\left(\boldsymbol{\Phi} \mathbf{Q}_{B}^{(k)}-\boldsymbol{\Phi}^{*} \mathbf{Q}_{B}^{*(k)}\right)
$$

Multiplying by $\boldsymbol{\Phi}^{* T} \mathbf{J}$, using $\boldsymbol{\mu}=\left[\lambda_{1}, \ldots, \lambda_{d}\right]$, this gives

$$
\mathbf{Q}^{(n)}=\boldsymbol{\mu}^{n-1} \mathbf{Q}^{(1)}+\sum_{k=1}^{n-1} \boldsymbol{\mu}^{n-k-1} \mathbf{Q}_{B}^{(k)}
$$

In the same way, from (9), one has

$$
\mathbf{Q}^{*(N+1)}=\boldsymbol{\mu}^{* N-n+1} \mathbf{Q}^{*(n)}+\sum_{k=n}^{N} \boldsymbol{\mu}^{* N-k} \mathbf{Q}_{B}^{*(k)}
$$

with $\boldsymbol{\mu}^{*}=\boldsymbol{\mu}^{-1}$. We get

$$
\mathbf{Q}^{*(n)}=\boldsymbol{\mu}^{N+1-n} \mathbf{Q}^{*(N+1)}-\sum_{k=n}^{N} \boldsymbol{\mu}^{k+1-n} \mathbf{Q}_{B}^{*(k)}
$$

If we call $\mathbf{R}^{\left(n_{s}\right)}$ the vector of reaction corresponding to the constrain $s$ placed in $n_{s}$, the state vector is given as follow using (11):

$$
\begin{aligned}
\mathbf{u}^{(n)}=\boldsymbol{\Phi} \boldsymbol{\mu}^{n-1} \mathbf{Q}^{(1)}-\boldsymbol{\Phi}^{*} \boldsymbol{\mu}^{N+1-n} & \mathbf{Q}^{*(N+1)}+\mathbf{T}^{(n)}+ \\
& -\boldsymbol{\Phi} \sum_{n_{s}<n} \boldsymbol{\mu}^{n-1-n_{s}} \boldsymbol{\Phi}_{q}^{* T} \mathbf{R}^{\left(n_{s}\right)}+\boldsymbol{\Phi}^{*} \sum_{n_{s} \geq n} \boldsymbol{\mu}^{n_{s}+1-n} \boldsymbol{\Phi}_{q}^{T} \mathbf{R}^{\left(n_{s}\right)}
\end{aligned}
$$

$\mathbf{T}^{(n)}$ gathers all the known terms obtained from $\mathbf{F}_{e x t}^{(k)}$ and $\mathbf{F}_{I}^{(k)}$. 


\subsection{Boundary conditions}

The application of the conditions can be written in a synthetic way at support $s$ as:

$$
\mathbf{L}_{s} \mathbf{u}^{\left(n_{s}\right)}=\mathbf{B}_{s}
$$

The matrix $\mathbf{L}_{s}$ is a logical matrix or $(0,1)$ matrix. It can assume different forms depending on the type of constrain and his position along the structure. For a one-dimensional beam and a fixed support, $\mathbf{L}_{s}$ is given by:

$$
\mathbf{L}_{s}=\left[\begin{array}{llllll}
1 & 0 & 0 & 0 & 0 & 0 \\
0 & 1 & 0 & 0 & 0 & 0 \\
0 & 0 & 1 & 0 & 0 & 0
\end{array}\right]
$$

The index matrix $\mathbf{L}_{s}$ for two-dimensional and three-dimensional structures is not so easy to find. The strategy used is to construct the matrix $\mathbf{L}_{s}$ by means of sub-matrices that subsequently can be assembled. For multiple node junctions, the column state vector is composed by the $d$ nodal displacements followed by the $d$ nodal forces. The Matrix $\mathbf{L}_{s}$, that multiplies the state vector (see equation (23)) can be seen as the composition of two square sub-matrices $\mathbf{L}_{q}$ and $\mathbf{L}_{F}$ that apply to displacements and forces respectively:

$$
\mathbf{L}_{s} \mathbf{u}^{\left(n_{s}\right)}=\left[\begin{array}{l|l}
\mathbf{L}_{q} & \mathbf{L}_{F}
\end{array}\right]\left[\begin{array}{c}
\{\mathbf{q}\} \\
\{\mathbf{F}\}
\end{array}\right]
$$

The matrices $\mathbf{L}_{q}$ and $\mathbf{L}_{F}$, in turn, consist of sub-matrices, corresponding to each node, named $\mathbf{L}_{q, s u b}$ and $\mathbf{L}_{F, s u b}$. These sub-matrices are square, identical for every node and depend on the kind of support. Their dimensions correspond to the number of dof for each node.

$$
\mathbf{L}_{q}=\left[\begin{array}{lll}
\mathbf{L}_{q, s u b} & & \\
& \ddots & \\
& & \mathbf{L}_{q, \text { sub }}
\end{array}\right], \mathbf{L}_{F}=\left[\begin{array}{lll}
\mathbf{L}_{F, s u b} & & \\
& \ddots & \\
& & \mathbf{L}_{F, \text { sub }}
\end{array}\right]
$$

The sub-matrices $\mathbf{L}_{q, s u b}$ and $\mathbf{L}_{F, \text { sub }}$ have to be constructed for every type of structure (beam, shell, plates, etc.) from whom the number of dof per node depends. The boundary conditions are denoted by the column vector $\mathbf{B}_{s}$, where the subscript refers to the respective constrain $s$. Each component represents the admitted displacement of each dof. The vector $\mathbf{B}_{s}$ depend on the type of constrain:

- rigid constrains: $\mathbf{B}_{s}=[\mathbf{0}]$

- elastic constrain: $\mathbf{B}_{s} \neq[\mathbf{0}]$

\subsection{Solution of the problem}

In order to apply the boundary conditions, equation (22) has to be multiplied for the index matrix $\mathbf{L}_{s}$ and equalled to the vector $\mathbf{B}_{s}$. For each support $s$, the boundary conditions can be written as $\mathbf{B}_{s}=\mathbf{L}_{s} \mathbf{u}^{\left(n_{s}\right)}$ so that:

$$
\begin{aligned}
\mathbf{L}_{s} \boldsymbol{\Phi} \boldsymbol{\mu}^{n_{s}-1} \mathbf{Q}^{(1)}- & \mathbf{L}_{s} \boldsymbol{\Phi}^{*} \boldsymbol{\mu}^{N+1-n_{s}} \mathbf{Q}^{*(N+1)}+\mathbf{L}_{s} \mathbf{T}^{\left(n_{s}\right)}+ \\
& -\mathbf{L}_{s} \boldsymbol{\Phi} \sum_{n_{i}<n_{s}} \boldsymbol{\mu}^{n_{s}-1-n_{i}} \boldsymbol{\Phi}_{q}^{* T} \mathbf{R}^{\left(n_{i}\right)}+\mathbf{L}_{s} \boldsymbol{\Phi}^{*} \sum_{n_{i} \geq n_{s}} \boldsymbol{\mu}^{n_{i}+1-n_{s}} \boldsymbol{\Phi}_{q}^{T} \mathbf{R}^{\left(n_{i}\right)}=\mathbf{B}_{s}
\end{aligned}
$$


The next step consists in replacing the vector $\mathbf{R}^{\left(n_{i}\right)}$ with the expression $\mathbf{L}_{i}^{T} \tilde{\mathbf{R}}^{\left(n_{i}\right)}$ in order to consider only the non zero components of the reaction. For a notation issue, the following assumption are made:

$$
\Phi_{s}=\mathbf{L}_{s} \Phi ; \quad \Phi_{s}^{*}=\mathbf{L}_{s} \Phi^{\star}
$$

Finally:

$$
\begin{aligned}
\boldsymbol{\Phi}_{s} \boldsymbol{\mu}^{n_{s}-1} \mathbf{Q}^{(1)}- & \boldsymbol{\Phi}_{s} \sum_{n_{i}<n_{s}} \boldsymbol{\mu}^{n_{s}-1-n_{i}} \boldsymbol{\Phi}_{i}^{* T} \tilde{\mathbf{R}}^{\left(n_{i}\right)} \\
& +\boldsymbol{\Phi}_{s}^{*} \sum_{n_{i} \geq n_{s}} \boldsymbol{\mu}^{n_{i}+1-n_{s}} \boldsymbol{\Phi}_{i}^{T} \tilde{\mathbf{R}}^{\left(n_{i}\right)}-\boldsymbol{\Phi}_{s}^{*} \boldsymbol{\mu}^{N+1-n_{s}} \mathbf{Q}^{*(N+1)}=\mathbf{B}_{s}-\mathbf{L}_{s} \mathbf{T}^{\left(n_{s}\right)}
\end{aligned}
$$

Equation (28) can be written for every constrain $s$, leading to a system of linear equations in the form:

$$
\mathrm{AX}=\mathbf{F}
$$

The vector of the unknowns is:

$$
\mathbf{X}=\left[\begin{array}{c}
\mathbf{Q}^{(1)} \\
\tilde{\mathbf{R}}^{\left(n_{1}\right)} \\
\vdots \\
\tilde{\mathbf{R}}^{\left(n_{s}\right)} \\
\vdots \\
\tilde{\mathbf{R}}^{\left(n_{S}\right)} \\
\mathbf{Q}^{*(N+1)}
\end{array}\right]
$$

Once matrix $\mathbf{A}$ and vector $\mathbf{F}$ are defined, it is possible to solve the problem:

$$
\mathbf{X}=\mathbf{A}^{-1} \mathbf{F}
$$

Replacing the reactions $\mathbf{R}$ and wave amplitudes $\mathbf{Q}^{(1)}$ and $\mathbf{Q}^{*(N+1)}$ in equation (22), the response of the entire periodic structure is obtained.

\section{APPLICATIONS}

In order to validate this technique, two applications have been developed. The two structures will present different dimensions and types of constraints placed in an arbitrary manner. For each application the frequency response function (FRF) of the structure will be calculated with respect to a specific point. To confirm the results, every problem will be solved both by the FE method and the WFE method. Furthermore, for both methods the calculation time will be given so that the efficiency can be compared. The general procedure consists in dividing the structure into a certain number of identical substructures. Using the software Abaqus, the substructure is modelled and the stiffness and mass matrix obtained. By importing this data, the problem can be solved by the software Matlab. In fact, from the stiffness and mass matrix it is possible to obtain the dynamic stiffness matrix and the transfer matrix $\mathbf{S}$.

\subsection{D Beam}

The structure considered is the multi supported beam represented in Figure (2) with $L=$ $50 \mathrm{~m}$. The objective of this application is to demonstrate the possibility of considering different types of constrained not necessarily equispaced. 


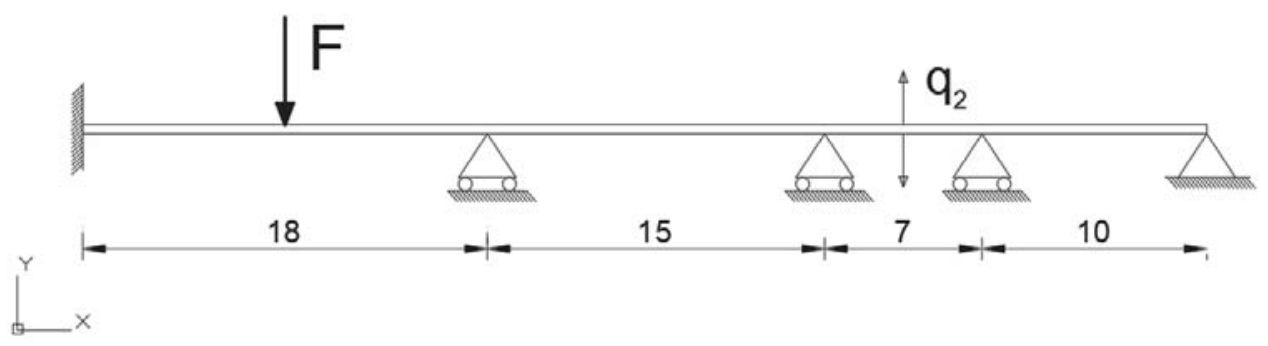

Figure 2: Multi-supported beam

The section of the beam is a IPE 400, see Figure (7) and Table (3). The external force is a vertical point force and acts to the middle of the first span. His value is $F=5 k N$. The material considered is steel and the mechanical properties are summarized in Table (1).

\begin{tabular}{ll}
\hline Steel & \\
\hline Density & $d=7850 \mathrm{~kg} / \mathrm{m}^{3}$ \\
Elastic modulus & $E=210 \mathrm{GPa}$ \\
Poisson modulus & $\nu=0.3$ \\
\hline
\end{tabular}

Table 1: Mechanical properties of steel.

The beam is composed by 220 substructures of length $l=0.2 \mathrm{~m}$ with 33 dofs by period and 6603 dofs in the whole structure. The type of element is a 2-node linear beam in a plane. In Figure (3) are reported the results of the analysis. The results match perfectly. The computational time is drastically reduced being $49.31 s$ for FEM and $5.57 s$ for WFEM.

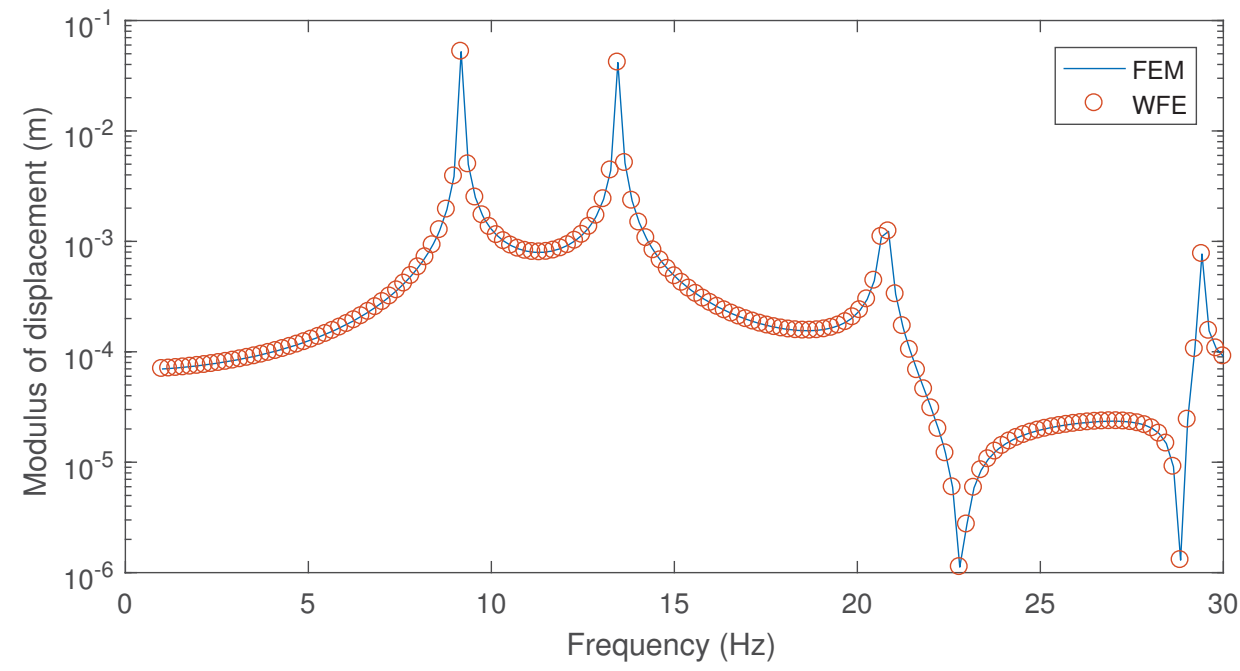

Figure 3: Frequency response function. Logarithm of the modulus of the displacement in function of the frequency.

\subsection{Multi-supported bridge}

In this section, the dynamic behaviour of a multi-supported bridge is studied. The total length is $L=120 \mathrm{~m}$. The bridge is fixed to the ends and supported by two equispaced roller supports. 
The maximum span is $L_{\text {span }}=40 \mathrm{~m}$. The longitudinal scheme of the bridge is represented in Figure (4). The bridge presents a box-beam deck which dimensions are represented in Figure

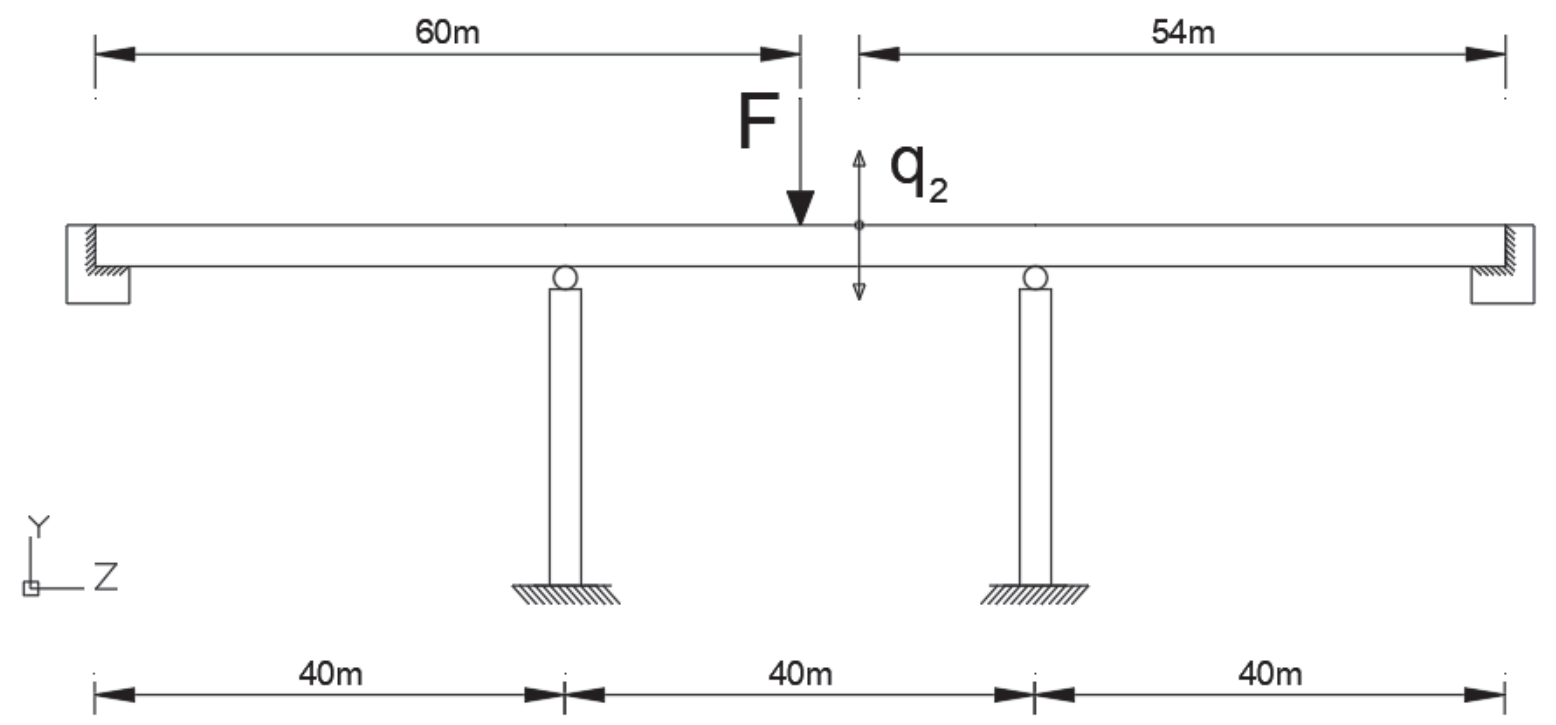

Figure 4: Multi-supported bridge

(5). The external force is a vertical point force and acts to the middle of the structure; its value

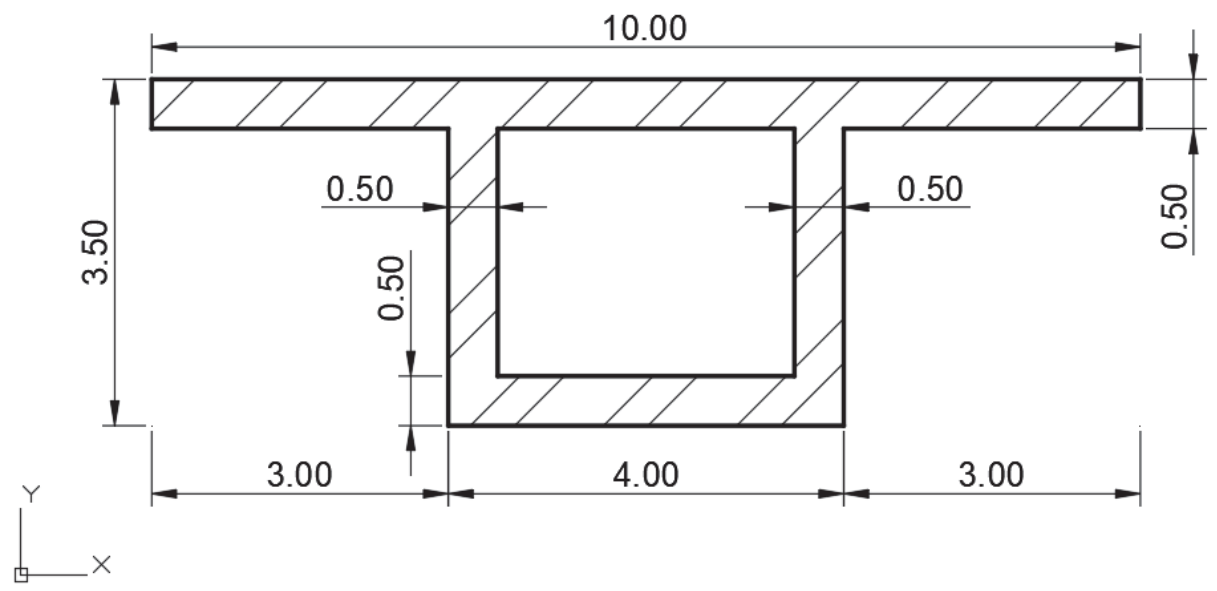

Figure 5: Section of the bridge $(m)$.

is $F=10 k N$. The position is at the right upper corner of Figure (5). The response is also computed at the right upper corner. The material considered is concrete and the mechanical property are summarized in Table (2).

The bridge is composed by 480 substructures of length $l=0.25 \mathrm{~m}$ with 1368 dofs by period and 329004 dofs in the whole structure. The substructure has been modelled by a 8-node linear brick of dimensions $0.25 \mathrm{~m}$. The FRF has been computed every $0.15 \mathrm{~Hz}$ in the range of frequencies $0-30 \mathrm{~Hz}$. The result obtained by applying the WFE method and showed in Figure (6) matches with the one obtained with the FEM. Moreover the computational time is halved being 204 minutes for FEM and 100 minutes for WFEM equivalent to $51 \%$ of time reduction. 


\begin{tabular}{ll}
\hline HP Concrete & \\
\hline Density & $d=2500 \mathrm{~kg} / \mathrm{m}^{3}$ \\
Elastic modulus & $E=48 \mathrm{GPa}$ \\
Poisson modulus & $\nu=0.2$ \\
\hline
\end{tabular}

Table 2: Physical and Mechanical characteristic of concrete.

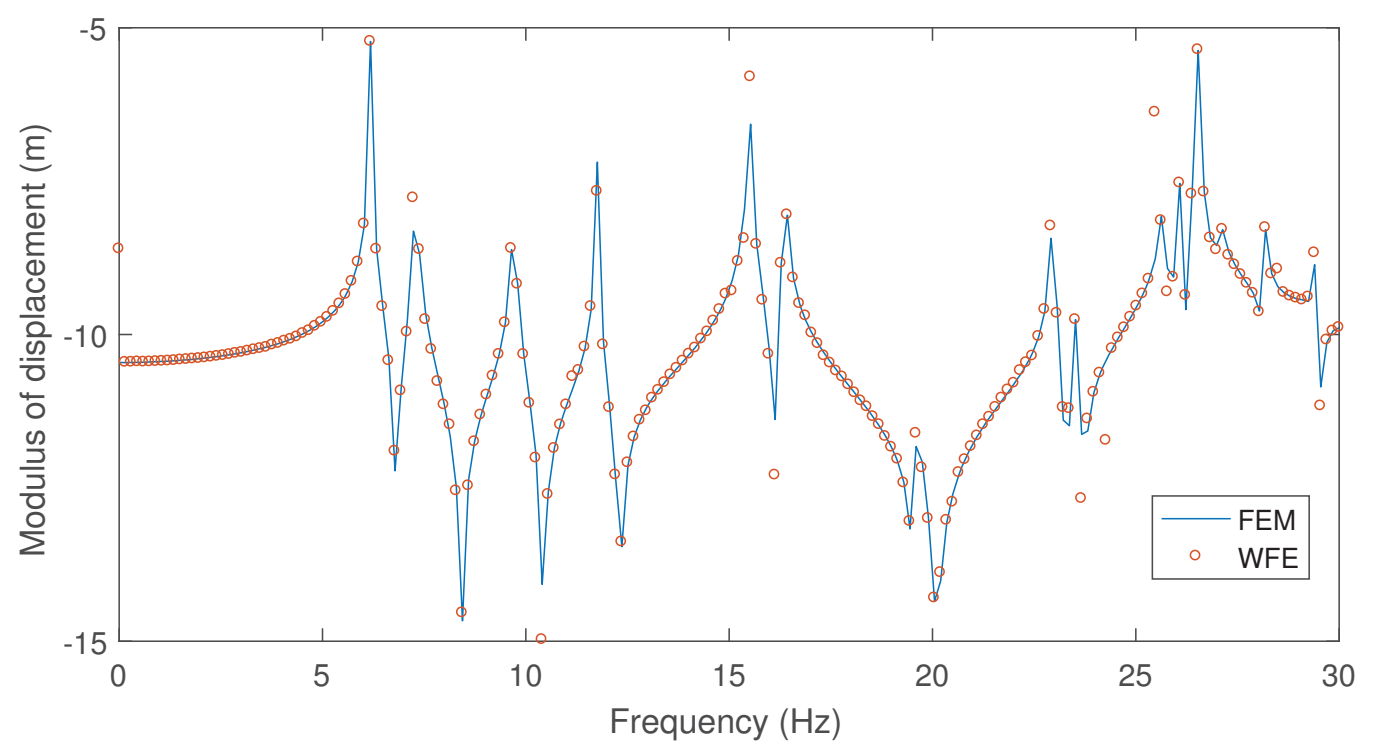

Figure 6: FRF of the bridge. Comparison between the WFEM (o) and the FEM (-)

\section{CONCLUSIONS}

As already mentioned, the WFE method has proved to be an excellent calculation tool to obtain results in a definitely shorter time compared to FEM. The proposed technique, that can be seen as a way to impose the boundary conditions, is simple but at the same time very effective. It is important to highlight the high efficiency in terms of time reduction and memory used during the computation. Finally, through practical applications, we have validated the method by finding results that coincide with the FEM but with the advantages of obtaining a computational time that in the worst case is halved.

\begin{tabular}{ll}
\hline IPE 400 & \\
\hline$h$ & $400 \mathrm{~mm}$ \\
$b$ & $180 \mathrm{~mm}$ \\
$t_{w}$ & $8.6 \mathrm{~mm}$ \\
$t_{f}$ & $\nu=13.5 \mathrm{~mm}$ \\
\hline
\end{tabular}

Table 3: Dimensions of IPE 400 section 


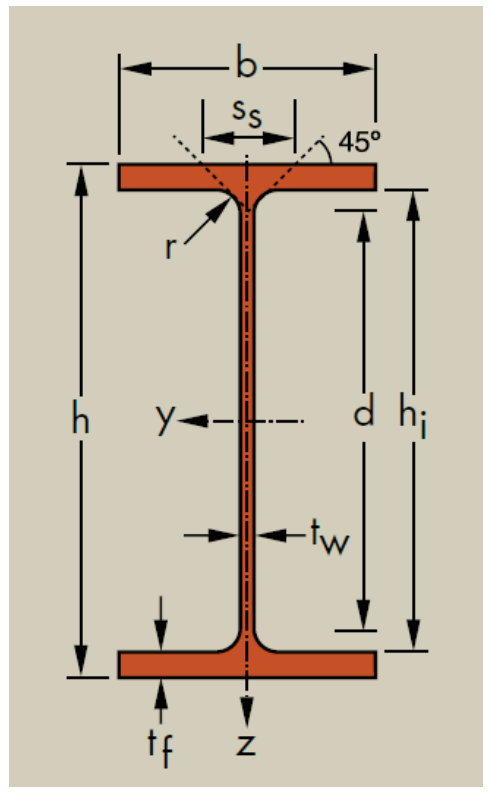

Figure 7: IPE 400

\section{REFERENCES}

[1] D. Duhamel, B.R. Mace, M.J. Brennan, Finite element analysis of the vibrations of waveguides and periodic structures, Journal of sound and vibration, 294(1-2), 205-220, 2006

[2] B. Mace, D. Duhamel, M. Brennan, L. Hinke, Finite element prediction of wave motion in structural waveguides, The Journal of the Acoustical Society of America, 117, 2835-43, 2005

[3] J.M. Mencik, D. Duhamel, A wave-based model reduction technique for the description of the dynamic behavior of periodic structures involving arbitrary-shaped substructures and large-sized finite element models, Finite Elements in Analysis and Design, 101, 1-14, 2015

[4] T. Hoang, D. Duhamel, G. Foret, Wave finite element method for vibration of periodic structures subjected to external loads, 6th European Conference on Computational Mechanics (ECCM 6), June 2018, Glasgow, United Kingdom

[5] T. Hoang, D. Duhamel, G. Foret, J.L Pochet, F. Sabatier, Wave finite element method and moving loads for the dynamic analysis of railway tracks, 13th World Congress on Computational Mechanics (WCCM XIII), Jul 2018, New York, United States 